\title{
Un ptosis unilatéral isolé
}

\section{An Isolated Unilateral Ptosis}

\author{
P. Parsis · C. Forestier · M. Martinez
}

Reçu le 25 janvier 2018; accepté le 2 mars 2018

(C) SFMU et Lavoisier SAS 2018

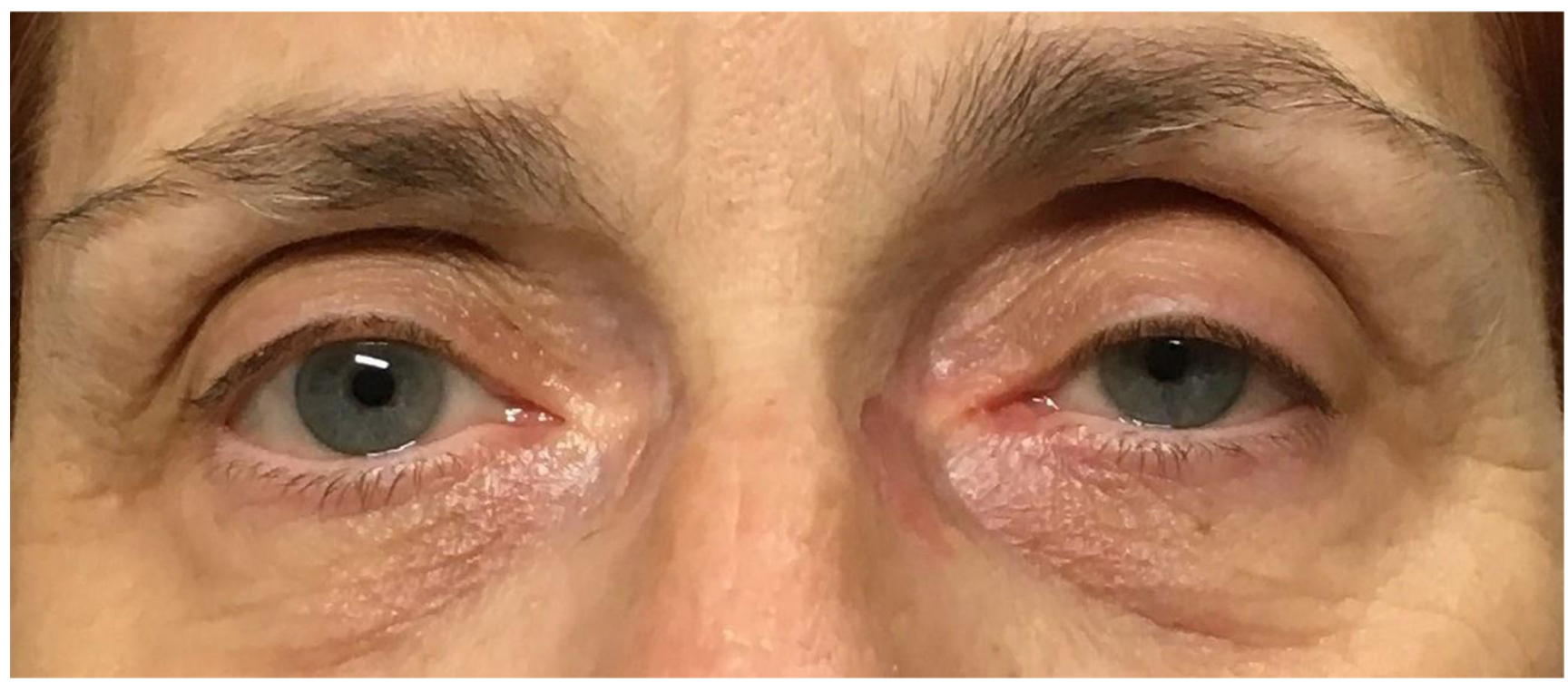

Fig. 1 Ptosis gauche isolé (avec accord de la patiente)

Une patiente de 71 ans aux antécédents de néoplasie mammaire en rémission depuis 15 ans se présente aux urgences pour un ptosis isolé de l'œil gauche fixe, évoluant depuis 48 heures (Fig. 1), associé à un épisode fugace de diplopie binoculaire verticale résolutive. Elle décrit aussi des cervicalgies postérieures depuis plusieurs semaines. L'examen clinique ne retrouve pas d'anomalies autres que ce ptosis en particulier sur le plan neurologique (absence de syndrome de Claude-Bernard-Horner ou de signes de focalisation), la glycémie capillaire est à $1,2 \mathrm{~g} / \mathrm{l}$. L'électrocardiogramme est en rythme sinusal. On évoque alors l'hypothèse d'un tableau d'accident vasculaire cérébral pontique incomplet ou d'une

P. Parsis · C. Forestier · M. Martinez $(\bowtie)$

Pôle d'urgences, centre hospitalier du Forez,

F-42605 Montbrison, France

e-mail : mikael.martinez@ch-forez.fr

\section{Martinez}

Réseau d'urgence ligérien Ardèche Nord (REULIAN), centre hospitalier Le Corbusier, F-42700 Firminy, France atteinte microvasculaire du nerf oculomoteur. Une imagerie par résonnance magnétique (IRM) cérébrale avec angioIRM du polygone de Willis et des troncs supra-aortiques est réalisée en urgence et élimine cette hypothèse. On évoque alors le diagnostic possible de myasthénie [1]. L'épreuve de Mary-Walker est négative : elle consiste à faire contracter plusieurs fois le poing du patient sous un garrot; à la levée du garrot : le ptosis doit apparaître [2]. Cependant, ce test aisément réalisable aux urgences, bien que spécifique, ne présente pas une grande sensibilité. La radiopulmonaire est sans anomalies. Une consultation de neurologie est demandée avec réalisation d'un électroneuromyogramme qui met en évidence un décrément significatif pour les couples facial-orbiculaires de la paupière gauche et le couple spinal-trapèze gauche. Un traitement d'épreuve par pyridostigmine améliore la symptomatologie. La recherche d'anticorps antirécepteur à l'acétylcholine (anti-RACh) est positive, confirmant le diagnostic de myasthénie.

La myasthénie est une maladie auto-immune avec une prévalence de 50 à 200 par million d'habitants. Il existe deux 
pics d'incidence : vers 30 ans avec prépondérance féminine, et après 65 ans sans différence de sexe. Elle est liée à la production d'anticorps anti-RACh parfois en lien avec un thymome. Elle se manifeste insidieusement ou brutalement, le plus souvent par une fatigabilité anormale à l'effort et dans 50 à $60 \%$ des cas par une atteinte oculomotrice initiale. Une symptomatologie initiale de tête tombante est plus fréquente chez le sujet âgé : les cervicalgies présentées par la patiente correspondaient à la forme initiale de cette symptomatologie et non à une atteinte sensitive. Le diagnostic est posé sur un trouble neurologique moteur pur fluctuant, associé à une fatigabilité. Un test d'épreuve aux anticholinestérasiques permettant une amélioration de la symptomatologie, la présence d'un décrément supérieur à $10 \%$, au minimum sur deux couples nerf-muscle, lors des stimulations répétitives à trois cycles/seconde en électroneuromyographie ainsi que la mise en évidence d'anticorps anti-RACh confirment le diagnostic. Le traitement est composé d'un traitement symptomatique par anticholinestérasique et d'un traitement immunomodulateur, corticostéroïdes et azathioprine. La thymectomie est indiquée en cas de thymome ou de myasthénie à anticorps anti-RACh positifs chez les patients de moins de 50 ans. L'immunothérapie (immunoglobulines intraveineuses, échanges plasmatiques) est réservée au traitement des poussées sévères [3].

Liens d'intérêts : les auteurs déclarent ne pas avoir de lien d'intérêt.

\section{Références}

1. Gilhus NE (2016) Myasthenia gravis. N Engl J Med 375:2570-81

2. Patten MB (1975) A hypothesis to account for the Mary Walker phenomenon. Ann Intern Med 82:411-5

3. Sanders DB, Wolfe GI, Benatar M, et al (2016) International consensus guidance for management of myasthenia gravis. Neurology $87: 419-25$ 